\title{
STUDY ON SHADOW DETECTION FROM HIGH- RESOLUTION SATELLITE IMAGES USING COLOR MODEL
}

\author{
Yasser Mostafa ${ }^{a^{*}}$, Beshoy Nady ${ }^{\mathrm{b}}$ \\ ${ }^{a}$ Civil Eng. Dept. Faculty of Engineering, Sohag University, Sohag, Egypt \\ ${ }^{b}$ Civil Eng. Dept., Faculty of Engineering, Assuit University, Assuit, Egypt
}

\begin{abstract}
Shadow detection is an important process for applications like classification, change detection, image interpretation, object detection, and recognition. The existence of shadow in satellite images reduce the amount of information that can be extracted and accordingly makes these applications more difficult or even impossible. Different color space is used to detect shadows based on $R, G$, and $B$ bands. This paper aims to represent an automatic approach for shadow detection from high-resolution satellite images. In this approach, a new index to highlight shadow areas based on $C_{1} C_{2} C_{3}$ color model is developed. A comparative study is carried out between the proposed index with a different photometric invariant color model, including IHS, HSV, YIQ, and $Y C_{b} C_{r}$ models over the ratio and single-band images. Then, an automatic thresholding method is applied in images histogram. The accuracy of the obtained results is evaluated in terms of visual comparisons and shadow detection accuracy assessments. Experimental results show that the proposed ratio method provides the best results for shadow detection. On the other hand, shallow water is still misclassified as a shadow.
\end{abstract}

(C) 2021 Published by Faculty of Engineering - Sohag University. DOI: 10.21608/SEJ.2021.155942.

Keywords: shadow detection; color model; threshold; and accuracy assessment.

\section{INTRODUCTION}

A shadow occurs when an object partially or totally occludes direct light from a source of illumination. It is not difficult for human interpreters to identify shadows in satellite images since the shadow is one of the fundamental elements in visual photo interpretation. Presence of shadow reduces the successful rate of object recognition, edge extraction, image matching, and change detection. On the other hand, it can produce a great deal of useful information about shape, relative position, surface character, and other characters of the object generating shadow [1].

Regarding shadow detection in high-resolution satellite images, two main approaches are reported in the previous literature, namely, the model-based and the property-based. The former method needs an accurate 3D model and complete knowledge of the sun orientation. However, since usually, such knowledge is not available, most of the detection algorithms are based on shadow properties methods. The property-based approaches make use of certain shadow properties in images, such as brightness, spectral characteristics, and geometry which are directly deduced from the image data information $[2,3]$. Because of their simplicity both in principle and implementation, the property-based approaches have been widely used in literature.

* Corresponding author: yasser79gm@gmail.com 
Many property-based methods are introduced for shadow detection. The two main factors that affect shadow extraction are the index selected from the employed color model and the thresholding method. Several color models were used by researchers for the property-based method of shadow detection. Gevers and Smeulders, [4] proposed $\mathrm{C}_{1} \mathrm{C}_{2} \mathrm{C}_{3}$ color model. The $\mathrm{C}_{3}$ layer is used for shadow detection [5-7]. Texture filter and region growing techniques are then applied to extract shadows. Tsai [8] compared the effectiveness of various ratio maps from different color models including HSI, HSV, HCV, YIQ, and $\mathrm{YC}_{\mathrm{b}} \mathrm{C}_{\mathrm{r}}$ models for shadow detection. Spectral ratio of [8] is improved by Chung et al. [9] through stretch the gap between the ratio values of shadow and non-shadow pixels. Experimental results show that the improved method provides more reliable and accurate shadow detection results than the algorithms proposed by [8]. Ma et al. [10] introduced a new index from the HSV color space named Normalized Saturation Value Difference Index (NSVDI) to detect shadows. The image pixel, which has a higher NSVDI than zero threshold value, is categorized as a shadow; otherwise, it is non-shadow. Three different indices suggested by Cai et al. [11] for shadow detection based on HSI color space which produced more accurate results than using HSI color space only. Mostafa and Abdelhafiz [12] analyzing the spectral features of objects in high-resolution satellite images and developed a new image index for shadow detection based on highlighting shadow areas in the first principal component using R, G, and B bands.

Some problems can be found in property-based methods like the misclassification between shadow and dark objects such as water. Water areas provide low reflectance; therefore, they are commonly classified as a shadow. This paper introduced an effective and simple approach for shadow detection in high-resolution satellite images based on $\mathrm{C}_{1} \mathrm{C}_{2} \mathrm{C}_{3}$ color model.

The rest of this paper is organized as follows. Section 2 demonstrates the study area and data used. Section 3 describe color spaces of special interest for shadow detection. In Section 4, the proposed approach is briefly described. Accuracy assessment of shadow detection, which is applied in the present study, is detailed in Section 5. Results and discussion are demonstrated through Section 6. Conclusions are given in Section 7.

\section{STUDY AREA AND DATA USED:}

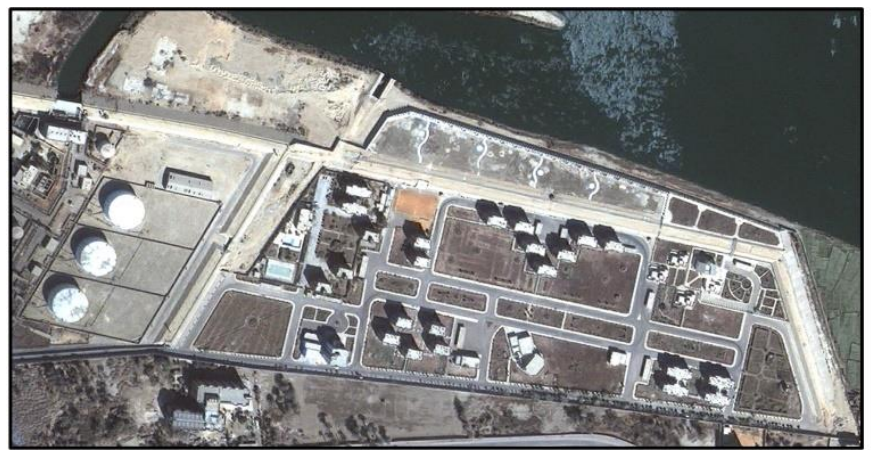

(a)

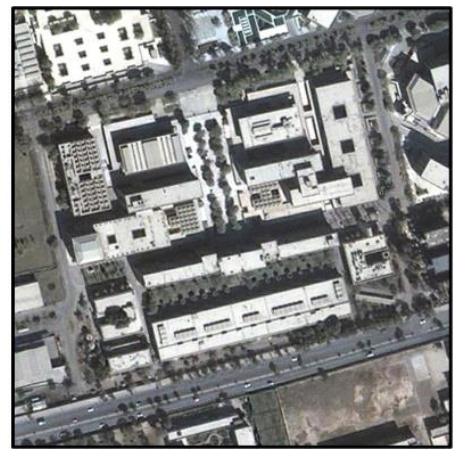

(b)

Fig. 1. RGB colour satellite images: (a) power station, (b) complex building.

Fig. 1 shows two study areas from pan sharpened IKONOS satellite image with a resolution of 1 meter. The first study area for power station Fig. 1(a) with a size of $951 \times 509 \mathrm{~m}$, includes water, bare soil, roads, buildings, diesel cisterns, trees, and so on. The second study area for complex building Fig. 1(b) with a size of $392 \times 394$ $\mathrm{m}$, includes buildings, roads, trees, and vehicles. The study areas were chosen with different land cover to examine the ability of the proposed method to deal with complex situations like water bodies and dark objects.

\section{INVARIANT COLOR SPACES:}

In visual interpretation applications the color tone is considered a powerful descriptor that simplifies and dominates feature identification. Color can be represented in a variety of three-dimensional spaces. Each color 
space is characterized by interesting properties which make it especially appropriated for a specific application. In the following, several color models are briefly described in terms of their relations with the RGB model.

\subsection{Intensity-Hue-Saturation (IHS) Color Space:}

Numerous algorithms have been developed and used to convert the RGB color space to the IHS color space [13]. In this study, the algorithm of RGB-to-IHS transformation, supported in the ERDAS software, has been adopted. Mostafa et al, [14] used IHS taking into consideration NIR1, RE, and CB bands instead of R, G, and B bands. The mathematical expressions described in the ERDAS field guide [15] as follows:

$$
\begin{aligned}
& \operatorname{Intensity}(I): \quad I=(M+m) / 2 \\
& \operatorname{Hue}(H)=\left\{\begin{aligned}
0, & \text { if } M=m \\
60(2+b-g), & \text { if } R=M \\
60(4+r-b), & \text { if } G=M
\end{aligned}\right. \\
& \text { Saturation }(S)=\left\{\begin{array}{c}
0, \quad \text { if } M=m \\
(M-m) /(M+m), \quad \text { if } I \leq 0.5 \\
(M-m) /(2-M+m), \quad \text { if } I>0.5
\end{array}\right.
\end{aligned}
$$

where $M$ is the largest value of $R, G$, and $B$ bands and $m$ is the least value of $R, G$, and $B$ bands. $r, g$, and $b \in$ $[0,1]$ are normalized bands of $R, G$, and $B$ bands, respectively.

\subsection{Hue-Saturation-Value (HSV) Color Space:}

In [16], Smith described a triangle based HSV model in the following relations with the RGB model:

$$
\begin{aligned}
& V=\frac{1}{3}(R+G+B) \\
& S=1-\frac{3}{R+G+B} \min (R, G, B) \\
& H=\left\{\begin{array}{r}
\theta, \text { if } B \leq G \\
360^{\circ}-\theta, \text { if } B>G
\end{array}\right.
\end{aligned}
$$

in which $\theta=\cos ^{-1}\left\{\frac{\frac{1}{2}[(R-G)+(R-B)]}{\sqrt{(R-G)^{2}+(R-B)(G-B)}}\right\}$.

\subsection{Luma-in phase-quadrature (YIQ) Color Space}

The YIQ color model is a widely supported standard in National Television Standards Commission (NTSC) color TV transmission. In this scheme, is proportional to the gamma-corrected luminance, which corresponds roughly with intensity, and jointly describes the chroma, which corresponds with hue and saturation, of a color image in the following relations with the RGB model:

$$
\left[\begin{array}{l}
Y \\
I \\
Q
\end{array}\right]=\left[\begin{array}{ccc}
0.299 & 0.587 & 0.114 \\
0.596 & -0.275 & -0.321 \\
0.212 & -0.523 & 0.311
\end{array}\right]\left[\begin{array}{l}
R \\
G \\
B
\end{array}\right]
$$




\subsection{Luminance - Chroma blue - Chroma red $\mathrm{YC}_{b} C_{r}$ Color Space:}

The $\mathrm{YC}_{\mathrm{b}} \mathrm{C}_{\mathrm{r}}$ model is used in most video and image compression standards like JPEG, MPEG, and H2.63+ for the transmission of luma and chroma components coded in the integer range [0, 255]. It has the following relations with the RGB model [17]:

$$
\left[\begin{array}{l}
Y \\
C_{b} \\
C_{r}
\end{array}\right]=\left[\begin{array}{ccc}
0.275 & 0.504 & 0.098 \\
-0.148 & -0.291 & 0.439 \\
0.439 & -0.368 & -0.071
\end{array}\right]\left[\begin{array}{c}
R \\
G \\
B
\end{array}\right]+\left[\begin{array}{c}
16 \\
128 \\
128
\end{array}\right]
$$

\subsection{First, Second, and Third chrominace $C_{1} C_{2} C_{3}$ Color Space}

The $C_{1} C_{2} C_{3}$ color space is a scale model between the three RGB components in RGB-space [18], the threecolor components of which vary with the surface reflection and the sensor. The model is defined as follows:

$$
\begin{aligned}
& C_{1}=\arctan \left(\frac{R}{\max (G, B)}\right) \\
& C_{2}=\arctan \left(\frac{G}{\max (R, B)}\right) \\
& C_{3}=\arctan \left(\frac{B}{\max (R, G)}\right)
\end{aligned}
$$

$C_{1} C_{2} C_{3}$-space, the best nonlinear transformation model for shadow detection, is barely sensitive to reflected light. Moreover, the brightness of the $\mathrm{R}$ and $\mathrm{G}$ color component is lower, but the $\mathrm{B}$ color component is larger than the non-shadow areas under the same reflection condition, so the $\mathrm{C}_{3}$ component is suitable for the shadow detection [19].

\section{THE PROPOSED APPROACH FOR SHADOW DETECTION ALGORITHM:}

Shadow detection indices aim to highlight the difference between shadow and non-shadow pixels in multispectral satellite images. Arévalo, et al [20] and Sarabandi et al [5] found that the $\mathrm{C}_{3}$ component is sensitive to shadow and can discriminate between shadows and other dark objects in the image. Jiang et al. [19] combined a normalized $\mathrm{C}_{3}$ component and a modified laplacian operator to detect shadows. However, the $\mathrm{C}_{3}$ component is unstable regarding to certain color values which leads to the misclassification of non-shadow pixels as a shadow [21]. This occurs for pixels with low saturation and those with extreme intensity (i.e., low and high, respectively). The proposed approach consists of two stages, which are the generation of a new image index to distinguish between shadow and non-shadow pixels as a first step. In the next step, the appropriate threshold is presented to automatically identify the shadow from the index.

\subsection{Ratio Shadow Index (RSI)}

As using a single-band image gives inaccurate detection of shadow pixels, it will be more convenient to use all the characteristics of the available bands of a multispectral image in the shadow detection process [22]. The satellite images are converted from RGB to $\mathrm{C}_{1} \mathrm{C}_{2} \mathrm{C}_{3}$ color model first. Then transformed components are scaled to the range of $[0,1]$ values. A ratio shadow index (RSI) is developed by utilizing $C_{1}$ and $C_{3}$ bands as follows:

$$
\mathrm{RSI}=\left(C_{3}+1\right) /\left(C_{1}+1\right)
$$

where $C_{1}$ and $C_{3}$ representing the first, and third chrominace in $\mathrm{C}_{1} \mathrm{C}_{2} \mathrm{C}_{3}$ color model, respectively. 


\subsection{Thresholding}

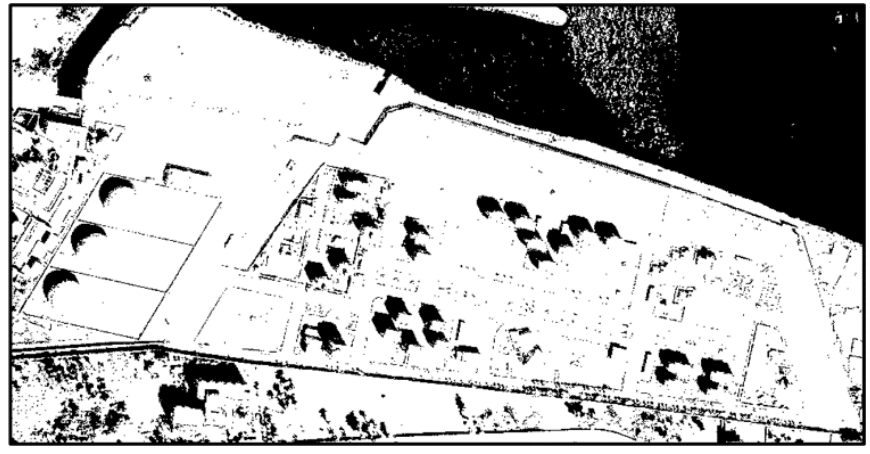

(a)

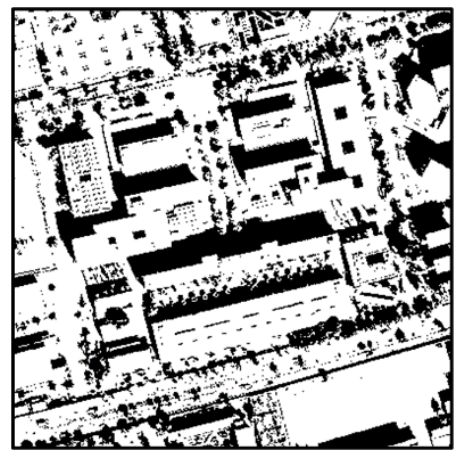

(b)

Fig. 2. Shadow mask with the proposed RSI (a) power station and (b) complex building.

When the automatic threshold is used, usually the whole sequence will be automatic. Otsu [23] proposed a histogram-based threshold selection method that is suitable for separating an object from its background. This method is used to automatically select the threshold $T$ to separate shadow regions from non-shadow regions in the intensity map. In Otsu's [23] method, a threshold $T$ is selected to maximize.

$$
V(T)=\frac{(\bar{\mu} \cdot \omega(T)-\mu(T))^{2}}{\omega(T) \cdot \mu(T)}
$$

where $\omega(T)=\sum_{i=0}^{T} P_{i}, \mu(T)=\sum_{i=T+1}^{255} P_{i}, \bar{\mu}=\sum_{i=o}^{255} i . P_{i}$ and $P_{i}$ is the probability of pixels with grey level $i$ in the image. Fig. 2 shows the application of the proposed index on the two study areas of Fig. 1.

\section{ACCURACY ASSESSMENT}

For accuracy assessment, true shadow images which are created by manual interpretations (Figs. 3 (a) and (b)) are used as reference images. The objective evaluation metrics are then computed for the proposed shadow images based on the concept of the error matrices [24] and the terminology in references [25, 26], the evaluation metrics of shadow detection accuracy assessment at the pixel level define as follows.

1) Producer's accuracies: (a) shadow: $\eta_{S}=\frac{T P}{T P+F N}$

$$
\text { (b) non - shadow: } \eta_{n}=\frac{T N}{F P+T N}
$$

2) User's accuracies: (a) shadow: $p_{s}=\frac{T P}{T P+F P}$

$$
\text { (b) non - shadow: } p_{n}=\frac{T N}{T P+F N}
$$

3) Overall accuracy: $\tau=\frac{T P+T N}{T P+T N+F P+F N}$

in which TP (True Positive) is the number of shadow pixels correctly identified, FN (False Negative) is the number of shadow pixels identified as non-shadows, FP (False Positive) is the number of non-shadow pixels identified as shadows, TN (True Negative) is the number of non-shadow pixels correctly identified, and TP + TN $+\mathrm{FP}+\mathrm{FN}$ stands for the total number of pixels in the image. 


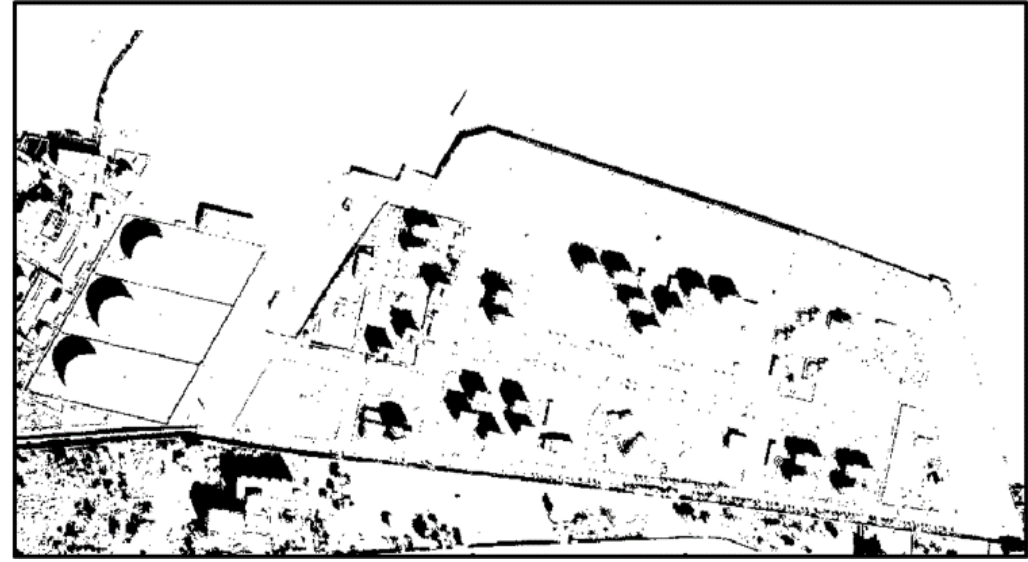

(a)

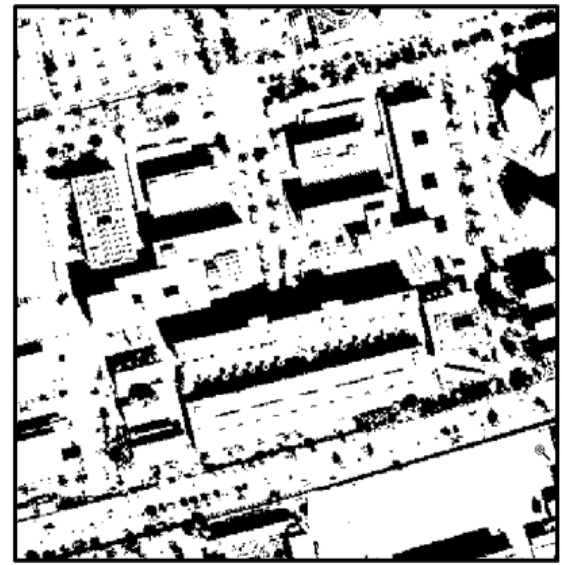

(b)

Fig. 3. Manually interpreted shadow masks (black area) as ground truth of shadow regions. (A) Shadow of the image in Figs. 1 (a) and (b) Shadow of the image in Fig. 1(b).

To validate the proposed RSI and check its efficiency, the obtained results are compared with effective singleband and the standard ratio color model mentioned in [8]. The two study areas are converted from RGB to four color models (IHS, HSV, YIQ, and $\mathrm{YC}_{\mathrm{b}} \mathrm{C}_{\mathrm{r}}$ ). Then transformed components are scaled to the range of $[0,1]$ values. The ratio maps are $(\mathrm{S}+1) /(\mathrm{I}+1)$ in IHS, $(\mathrm{H}+1) /(\mathrm{V}+1)$ in $\mathrm{HSV},(\mathrm{Q}+1) /(\mathrm{Y}+1)$ in $\mathrm{YIQ}$, and $\left(\mathrm{C}_{\mathrm{b}}+1\right) /(\mathrm{Y}+1)$ in $\mathrm{YC}_{\mathrm{b}} \mathrm{C}_{\mathrm{r}}$. The thresholding techniques are applied over the ratio and single-band ( $\mathrm{S}$ in IHS, H in HSV, Q in YIQ, $\mathrm{C}_{\mathrm{b}}$ in $\mathrm{YC}_{\mathrm{b}} \mathrm{C}_{\mathrm{r}}$, and $\mathrm{C}_{3}$ in $C_{1} C_{2} C_{3}$ ) images to delineate shadow regions. The images obtained will be binary images with all shadow pixels set to 0 and all non-shadow pixels are set to 1 .

TABLE 1: SHADOW DETECTION ACCURACY MEASUREMENTS OF THE IMAGE IN FIG. 1(A).

\begin{tabular}{|c|c|c|c|c|c|c|c|}
\hline \multirow{2}{*}{ Method } & \multirow[b]{2}{*}{$\begin{array}{l}\text { Colour } \\
\text { Model }\end{array}$} & \multirow{2}{*}{$\begin{array}{c}\text { Threshold } \\
\text { value }\end{array}$} & \multicolumn{2}{|c|}{ Producer's Accuracy } & \multicolumn{2}{|c|}{ User's Accuracy } & \multirow{2}{*}{$\begin{array}{c}\text { Overall Accuracy } \\
\tau(\%)\end{array}$} \\
\hline & & & $\begin{array}{l}\text { Shadow } \\
\eta_{s}(\%)\end{array}$ & $\begin{array}{c}\text { Non-shadow } \\
\eta_{n}(\%)\end{array}$ & $\begin{array}{l}\text { Shadow } \\
p_{s}(\%)\end{array}$ & $\begin{array}{c}\text { Non-shadow } \\
p_{n}(\%)\end{array}$ & \\
\hline \multirow{2}{*}{ IHS } & ratio & 117 & 98.45 & 69.79 & 25.24 & 99.77 & 72.48 \\
\hline & $\mathrm{S}$ & 93 & 97.20 & 71.94 & 26.41 & 99.60 & 74.31 \\
\hline \multirow{2}{*}{ HSV } & ratio & 111 & 99.76 & 59.57 & 20.35 & 99.96 & 63.34 \\
\hline & $\mathrm{H}$ & 85 & 99.84 & 59.54 & 20.36 & 99.97 & 63.32 \\
\hline \multirow{2}{*}{ YIQ } & ratio & 172 & 99.74 & 45.03 & 15.82 & 99.94 & 50.17 \\
\hline & Q & 182 & 99.72 & 36.95 & 14.08 & 99.92 & 42.84 \\
\hline \multirow{2}{*}{$\mathrm{YC}_{\mathrm{b}} \mathrm{C}_{\mathrm{r}}$} & ratio & 134 & 99.86 & 57.91 & 19.73 & 99.98 & 61.85 \\
\hline & $\mathrm{C}_{\mathrm{b}}$ & 186 & 99.95 & 41.17 & 14.97 & 99.99 & 46.69 \\
\hline \multirow{2}{*}{$\mathrm{C}_{1} \mathrm{C}_{2} \mathrm{C}_{3}$} & RSI & 66 & 86.90 & 75.38 & 26.77 & 98.23 & 76.46 \\
\hline & $\mathrm{C}_{3}$ & 115 & 99.50 & 59.93 & 20.46 & 99.91 & 63.64 \\
\hline
\end{tabular}

Shadow detection is evaluated in terms of visual comparisons and accuracy assessments. Shadow masks are compared with the true shadow masks of Figs. 3(a) and (b), respectively. Accuracy measurements results of shadow detection of the power station Fig. 1(a) and the complex building Fig. 1(b) are summarized in Tables 1 and 2, respectively. Fig. 4 and Fig. 5 show shadow extraction results of the ratio and single-band images, respectively. 
TABLE 2: SHADOW DETECTION ACCURACY MEASUREMENTS OF THE IMAGE IN FIG. 1(B).

\begin{tabular}{|c|c|c|c|c|c|c|c|}
\hline \multirow{2}{*}{ Method } & \multirow[b]{2}{*}{ Color Model } & \multirow{2}{*}{$\begin{array}{c}\text { Threshold } \\
\text { value }\end{array}$} & \multicolumn{2}{|c|}{ Producer's Accuracy } & \multicolumn{2}{|c|}{ User's Accuracy } & \multirow{2}{*}{$\begin{array}{l}\text { Producer's Accuracy } \\
\text { Shadow } \eta_{s}(\%)\end{array}$} \\
\hline & & & $\begin{array}{c}\text { Shadow } \\
\eta_{s}(\%)\end{array}$ & $\begin{array}{c}\text { Non-shadow } \\
\eta_{n}(\%)\end{array}$ & $\begin{array}{c}\text { Shadow } \\
\eta_{s}(\%)\end{array}$ & $\begin{array}{c}\text { Non-shadow } \\
\eta_{n}(\%)\end{array}$ & \\
\hline \multirow{2}{*}{ IHS } & ratio & 123 & 100.00 & 63.85 & 50.31 & 100.00 & 73.53 \\
\hline & $\mathrm{S}$ & 85 & 99.76 & 87.81 & 74.97 & 99.90 & 91.01 \\
\hline \multirow{2}{*}{ HSV } & ratio & 112 & 100.00 & 70.54 & 55.40 & 100.00 & 78.43 \\
\hline & $\mathrm{H}$ & 120 & 100.00 & 68.77 & 53.95 & 100.00 & 77.14 \\
\hline \multirow{2}{*}{ YIQ } & ratio & 124 & 100.00 & 51.33 & 42.92 & 100.00 & 64.37 \\
\hline & $\mathrm{Q}$ & 149 & 99.78 & 43.89 & 39.42 & 99.82 & 58.86 \\
\hline \multirow{2}{*}{$\mathrm{YC}_{\mathrm{b}} \mathrm{C}_{\mathrm{r}}$} & ratio & 131 & 100.00 & 47.13 & 40.91 & 100.00 & 61.30 \\
\hline & $\mathrm{C}_{\mathrm{b}}$ & 160 & 100.00 & 45.28 & 40.07 & 100.00 & 59.94 \\
\hline \multirow{2}{*}{$\mathrm{C}_{1} \mathrm{C}_{2} \mathrm{C}_{3}$} & RSI & 58 & 98.71 & 92.74 & 83.26 & 99.50 & 94.34 \\
\hline & $\mathrm{C}_{3}$ & 101 & 99.86 & 76.92 & 61.29 & 99.94 & 83.07 \\
\hline
\end{tabular}

\begin{tabular}{|c|c|c|}
\hline $\begin{array}{l}\text { Color } \\
\text { model }\end{array}$ & $\begin{array}{c}\text { Shadow mask of power station in } \\
\text { Figure (1-A) }\end{array}$ & $\begin{array}{c}\text { Shadow mask of complex } \\
\text { buildingin Figure(1-B) }\end{array}$ \\
\hline $\begin{array}{l}\text { IHS: } \\
\frac{S+1}{I+1}\end{array}$ & 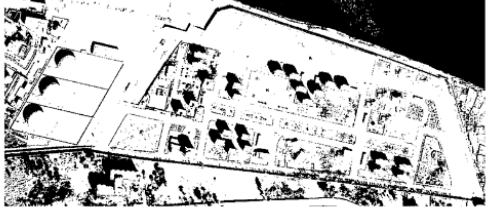 & dPas \\
\hline $\begin{array}{l}\text { HSV: } \\
\frac{H+1}{V+1}\end{array}$ & 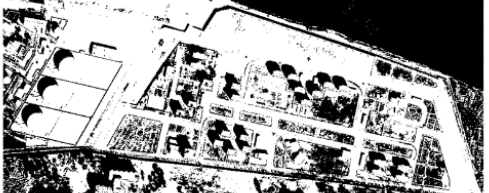 & 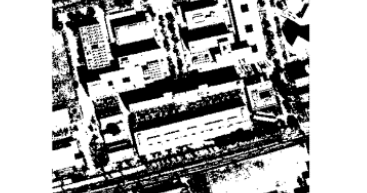 \\
\hline $\begin{array}{l}\text { YIQ: } \\
\frac{Q+1}{Y+1}\end{array}$ & & 5 \\
\hline $\begin{array}{l}\mathrm{YC}_{\mathrm{b}} \mathrm{C}_{\mathrm{r}}: \\
\frac{C_{b}+1}{Y+1}\end{array}$ & & 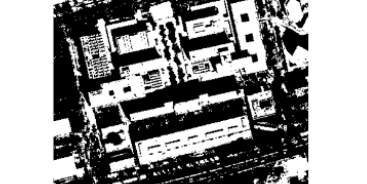 \\
\hline
\end{tabular}

Fig. 4. Shadow extraction (in black) results using ratio method. 


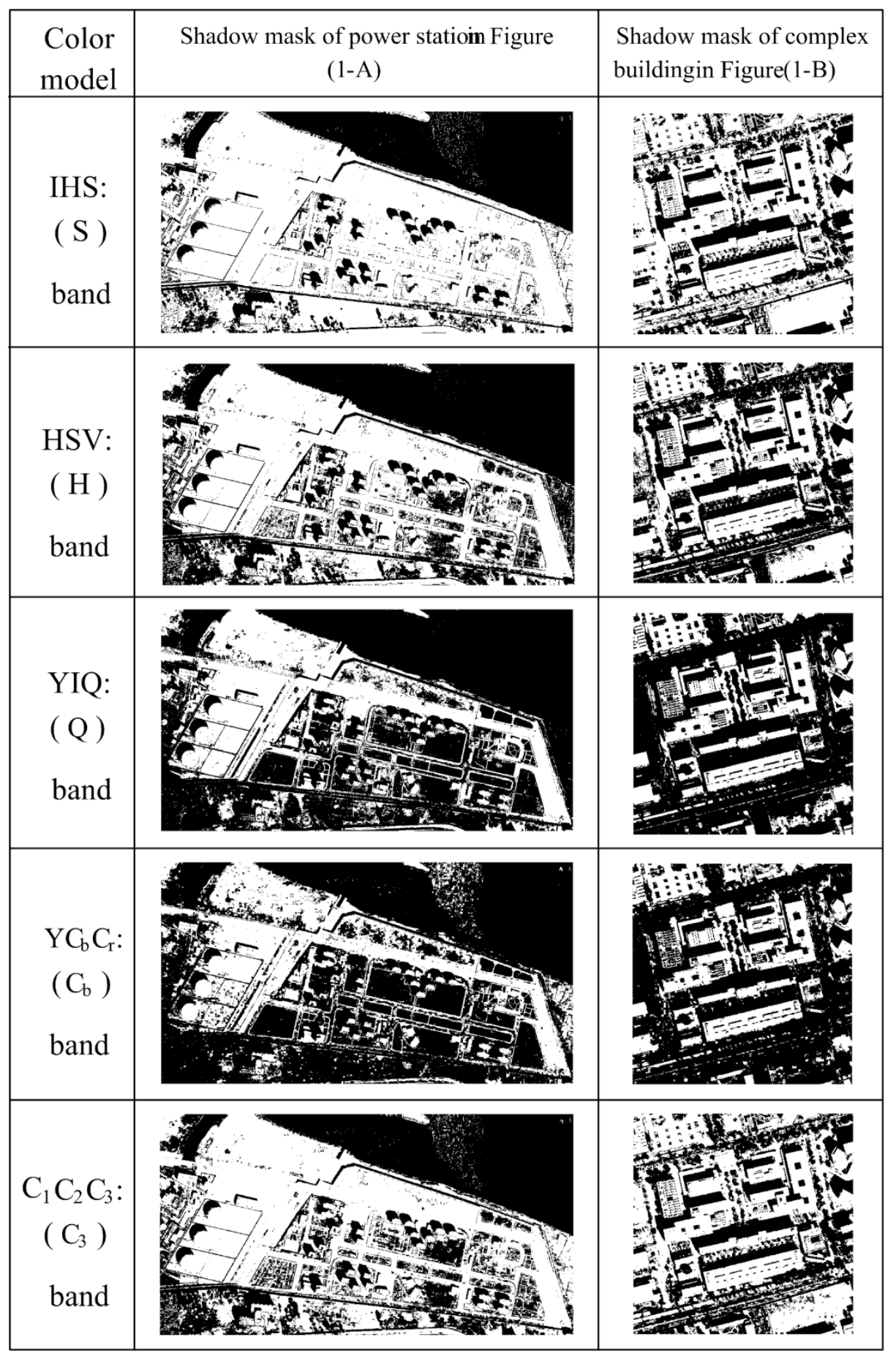

Fig. 5. Shadow extraction (in black) results from using single-band method.

\section{RESULTS AND DISCUSSION}

The proposed index RSI produces the highest overall accuracy results. Also, it gives better results than C3 band method. This can be attributed to the misclassification of small areas of bare soil and roads in $\mathrm{C} 3$ band as a shadow. The index gives an overall accuracy of $76.46 \%$ for study area A and $94.34 \%$ for study area B. Visual inspection of images in the ratio maps and single bands (Fig. 4 and Fig. 5) respectively, show that the differences 
between color models are variant. In the power station, the misclassification of shadow results from a large area of shallow water in the upper right corner reduces results in all color models. In IHS and HSV model, the same results are acquired in the ratio and single-band images as shown in Fig. 6. Where, the ratio method gives better results in YIQ and YCbCr models than single-band method. This is due to the misclassification of bare soil as shadow. In the complex building, single-band method gives better results in the IHS model ( $\mathrm{S}$ band) than the ratio method. The main reason is the misclassification of bare soil and roads as shadow in the ratio method. Where, in YIQ and YCbCr models the same results are acquired in the ratio and single-band images.

Overall accuracy

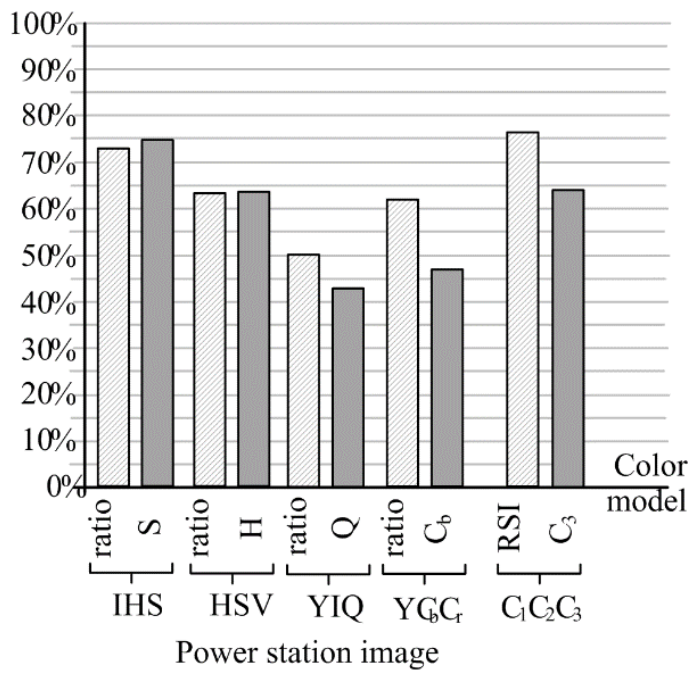

Overall accuracy

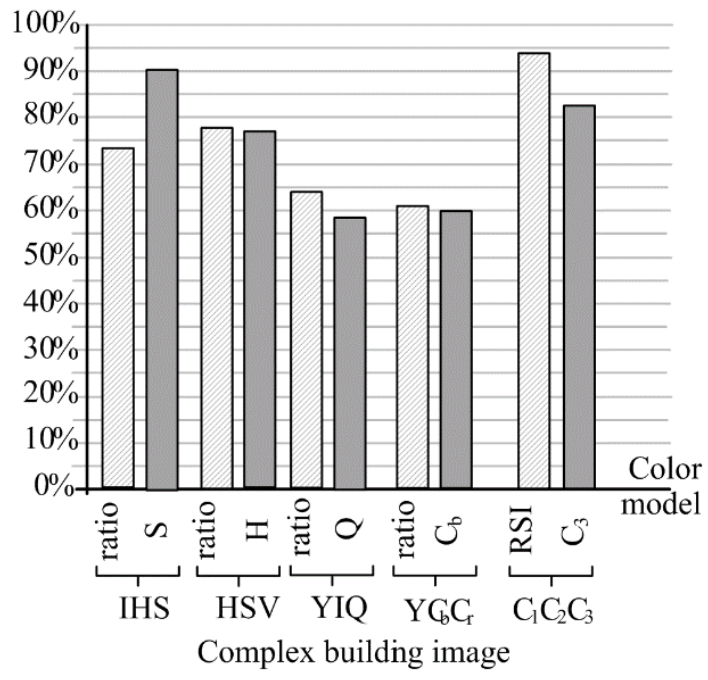

Fig. 6. Overall accuracy of shadow extraction results.

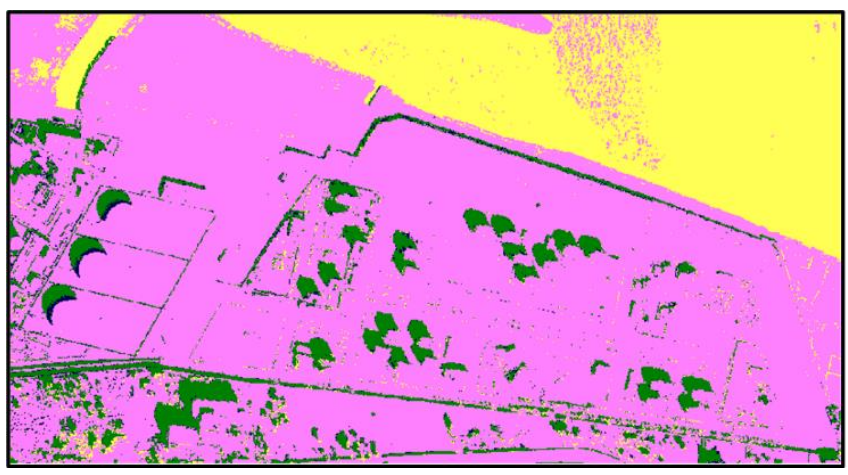

(a)

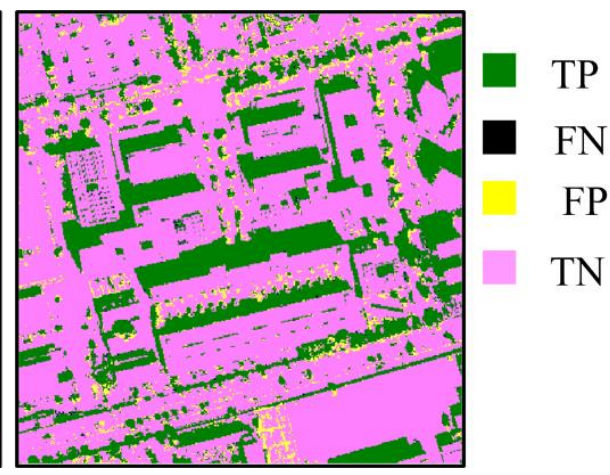

(b)

Fig. 7. Shadow extraction results of RSI.

Fig. 7 shows the results of shadow extracted by the proposed RSI. In this figure, shadow masks from RSI are superimposed on the true shadow masks of Figs. 2(a) and (b), respectively. The green color indicates areas of shadow that are classified correctly (TP). The black color indicates shadow areas that are misclassified as nonshadow (FN). This type of misclassification caused mainly by self-shadow as shown with diesel cisterns in the power station image. The yellow color indicates areas of non-shadow that are misclassified as a shadow (FP). The main yellow area in the power station consists of shallow water and small areas from dark vegetation. Where in complex buildings the main yellow area consists of dark vegetation and a small area from bare soil. The pink color indicates areas of non-shadow that are classified correctly (TN). 


\section{CONCLUSION}

In this work, a new shadow detection index is presented and validated. $C_{1}$ and $C_{3}$ bands from color model $\mathrm{C}_{1} \mathrm{C}_{2} \mathrm{C}_{3}$ are employed to form the RSI. Shadow pixels are then extracted from the index histogram using a certain threshold. The developed index was applied to two study areas and accuracy results are compared with ratio maps and single band. The proposed index achieved the highest accuracy. The experimental results demonstrate the effectiveness and stability of the proposed index. However, there is misclassification between dark object and water in the proposed approach since they are in the same side of the image histogram. It is recommended to separate dark objects such as water by using different methods before shadow detection.

\section{REFERENCES}

[1] Y. Mostafa and M. Abdelwahab, "Corresponding regions for shadow restoration in satellite high-resolution images," International Journal of Remote Sensing, vol. 39, no. 20, pp. 7014-7028, 2018.

[2] A. Movia, A. Beinat, and F. Crosilla, "Comparison of unsupervised vegetation classification methods from VHR images after shadows removal by innovative algorithms," The International Archives of the Photogrammetry, Remote Sensing and Spatial Information Sciences, vol. XL-7/W3, pp. 1269 - 1276, 2015.

[3] Y. Mostafa, "A review on various shadow detection and compensation techniques in remote sensing images," Canadian Journal of Remote Sensing, vol. 43, no. 6, pp. 545-562, 2017.

[4] T. Gevers and A. Smeulders, "Color-based object recognition," Pattern Recognition, vol. 32, no. 3, pp. 453-464, 1999.

[5] P. Sarabandi, F. Yamazaki, M. Matsuoka, and K. Kiremidjian, "Shadow detection and radiometric restoration in satellite high resolution images," In Proceedings of IEEE International Geoscience and Remote Sensing Symposium (IGARSS '04), Anchorage, AK, USA, 20-24 September 2004; vol 6: pp. 3744-3747, 2004.

[6] V. Arevalo, J. Gonzalez, J. Valdes, and G. Ambrosio, "Detecting shadows in Quickbird satellite images," ISPRS Commission VII Mid-term Symposium -Remote Sensing: From Pixels to Processes- Enschede, the Netherlands. pp. 330-335, 2006.

[7] Y. Mostafa and A. Abdelhafiz, "Shadow identification in high resolution satellite images in the presence of water regions," Photogrammetric Engineering and Remote Sensing, vol. 83, no. 2, pp. 87-94, 2017a.

[8] V. Tsai, "A comparative study on shadow compensation of color aerial images in invariant color models," IEEE Transactions on Geoscience and Remote Sensing, vol. 44, no. 6, pp. 1661 - 1671, 2006.

[9] K. L. Chung, Y. R. Lin, and Y. H. Huang, "Efficient shadow detection of color aerial images based on successive thresholding scheme," IEEE Transactions on Geoscience and Remote Sensing, vol. 47, no. 2, pp. 671-682, 2009.

[10] H. Ma, Q. Qin, and X. Shen, "Shadow segmentation and compensation in high resolution satellite images," IEEE International Geoscience and Remote Sensing Symposium, 2: 1036-1039, 2008.

[11] D. Cai, M. Li, Z. Bao, Z. Chen, W. Wei, and H. Zhang, "Study on shadow detection method on high resolution remote sensing image based on HIS space transformation and NDVI index," 18th International Conference on Geoinformatics,. Beijing: IEEE. pp. 1-4, 2010.

[12] Y. Mostafa and A. Abdelhafiz, "Accurate shadow detection from high resolution satellite images," IEEE Geoscience and Remote Sensing Letter, vol. 14, no. 4, pp. 494-498, 2017 b.

[13] N. Koutsias, M. Karteris, and E. Chuvieco, "The use of intensity-hue-saturation transformation of Landsat-5 thematic mapper data for burned land mapping," Photogrammetric Engineering and Remote Sensing, vol. 66, no. 7, pp. 829-839, 2000 .

[14] Y. Mostafa, M. Yousef, and F. Mostafa, "A new shadow detection index for 8-band very high-resolution satellite images," International Journal of Remote Sensing, vol. 41, no. 2, pp. 420-432, 2020.

[15] ERDAS, "Erdas Field Guide 5th ed ," Atlanta, Georgia: Erdas Inc, 2013.

[16] A. Smith, "Color gamut transform pairs," in Proc. SIGRAPH, vol. 3(ACM), Atalanta, GA, Aug. 23-25, pp. 12-19, 1978.

[17] P. Kumar, K. Sengupta, and A. Lee, "A comparative study of different color spaces for foreground and shadow detection for traffic monitoring system," in Proc. IEEE 5th Int. Conf. Intelligent Transportation Systems, Singapore, Sep. 2-6, pp. 100-105, 2002.

[18] [S. Elena, C. Andrea, and E. Touradj, "Shadow identification and classification using invariant color models," IEEE International Conference on Acoustics, Speech and Signal Processing, vol. 3: 1545-1548, 2001.

[19] J. Jiang, Y. Tian, M. Wang, Y. Zheng, and T. Yu, "A shadow detection algorithm for remote sensing images," Journal of Computational Information Systems, vol, 9: pp. 3783-3790, 2013.

[20] V. Arévalo, J. González, and G. Ambrosio, "Shadow detection in colour high-resolution satellite images," International Journal of Remote Sensing, vol. 29, no. 7, pp. 1945-1963, 2008.

[21] A. Shahtahmassebi, N. Yang, K. Wang, N. Moore, and Z. Shen, "Review of shadow detection and de-shadowing methods in remote sensing," Chinese geographical science, vol. 23, no. 4, pp. 403-420, 2013.

[22] S. Wang, and Y. Wang, "Shadow detection and compensation in high resolution satellite image based on retinex," in Proc. 5th Int. Conf. Image Graph., Xi'an, China, pp. 209-212, 2009. 
[23] N. Otsu, "A threshold selection method from gray level histograms," IEEE Trans. Syst., Man, Cybern., vol. SMC-9, no. 1, pp. 62-69, Mar, 1979.

[24] M. Lillesand, and W. Kiefer, "Remote Sensing and Image Interpretation," 4th ed. New York: Wiley, 2000.

[25] A. Prati, I. Mikic, M. Trivedi, and R. Cucchiara, "Detecting moving shadows: algorithms and evaluations," IEEE Trans. Pattern Anal. Mach. Intell., vol. 25, no. 7, pp. 918-923, Jul, 2003.

[26] J. Yao, and Z. Zhang, "systematic static shadow detection," in Proc. 17th Int. Conf. Pattern Recognition, vol. 2, pp. 76$79,2004$. 